\title{
Are temporal concepts embodied? A challenge for cognitive neuroscience
}

\author{
Alexander Kranjec* and Anjan Chatterjee \\ Department of Neurology, Center for Cognitive Neuroscience, University of Pennsylvania, Philadelphia, PA, USA
}

\section{Edited by:}

Diane Pecher, Erasmus University

Rotterdam, Netherlands

Reviewed by:

Max Louwerse, University of

Memphis, USA

Seana Coulson, University of California

at San Diego, USA

${ }^{*}$ Correspondence:

Alexander Kranjec, Department of Neurology, University of Pennsylvania,

3 West Gates Building, 3400 Spruce

Street, Philadelphia, PA 19104, USA.

e-mail:akranjec@mail.med.upenn.edu
Is time an embodied concept? People often talk and think about temporal concepts in terms of space. This observation, along with linguistic and experimental behavioral data documenting a close conceptual relation between space and time, is often interpreted as evidence that temporal concepts are embodied. However, there is little neural data supporting the idea that our temporal concepts are grounded in sensorimotor representations. This lack of evidence may be because it is still unclear how an embodied concept of time should be expressed in the brain. The present paper sets out to characterize the kinds of evidence that would support or challenge embodied accounts of time. Of main interest are theoretical issues concerning (1) whether space, as a mediating concept for time, is itself best understood as embodied and (2) whether embodied theories should attempt to bypass space by investigating temporal conceptual grounding in neural systems that instantiate time perception.

\section{Keywords: embodiment, abstract concepts, time and space, schemas}

\section{INTRODUCTION}

Time is frequently talked about in terms of space (Clark, 1973; Hasplemath, 1997; Evans, 2004; Tenbrink, 2007). For example, languages refer to the related temporal concepts of past, present, and future in spatial terms. Languages commonly conceptualize the past as behind, the future as in front of, and the present as here or co-locational with the space around the body. Behavioral data suggest that such conventions in language are not arbitrary; conceptual relations between space and time seem to reflect a psychological reality that is more than "language deep." That is, time appears to be thought about as well as talked about in terms of space (Boroditsky, 2000, 2001; Gentner and Boroditsky, 2001; Boroditsky and Ramscar, 2002; Matlock et al., 2005; Kranjec, 2006; Nunez and Sweetser, 2006; Nunez et al., 2006; Torralbo et al., 2006; Santiago et al., 2007; Casasanto and Boroditsky, 2008; Kranjec et al., 2010; Kranjec and McDonough, 2011).

Along these lines, some accounts of the data make the further claim that the tight coupling observed between these two domains supports an embodied view of cognition. Embodied cognition theories vary in their details, but most evoke a simulation of the concept under consideration (Wilson, 2002). They typically maintain that abstract concepts are given structure in the mind by the constraints and experiential couplings imposed by human physiology, as reflected in perception and action. Thus, for example, we may talk and think about past times as being "behind us" because, when we walk, what has already been experienced in time tends also to be located behind us in space.

Generally speaking, neuroscience research that investigates relations between temporal concepts and spatial semantics is limited (Kemmerer, 2005; Teuscher et al., 2008). Despite the wealth of linguistic and behavioral evidence, there is surprisingly little neural data supporting the idea that our temporal concepts are grounded in space, or more generally embodied in sensorimotor or perceptual representations. This lack of data is partly because it is unclear what such supportive evidence should look like in the first place.
Although the details of individual models differ, simulation accounts of embodied cognition (Barsalou, 1999; Glenberg and Kaschak, 2002; Zwaan, 2004) suggest that concepts activate the same perceptual and sensorimotor neural networks that represent actual experience with their real-world referents. Such explanations are intuitively plausible when accounting for the conceptualization of relatively concrete categories of objects and actions. For example, according to simulation theories, comprehending the word punch might be expected to activate sensorimotor representations associated with arms more than legs, whereas processing a word like kick might be expected to do the opposite. Results generally compatible with such predictions have been found (Buccino et al., 2005; Pulvermuller et al., 2005; Kemmerer et al., 2008) although questions about whether the precise details of the findings confirm the embodied hypothesis have been raised, and knowing how to best interpret the results from such studies is often unclear (Mahon and Caramazza, 2008; Chatterjee, 2010; Arévalo et al., 2010).

A more substantial problem for embodied theories concerns the representation of abstract concepts that are less obviously accessible to perception or direct experience. What does it mean to make claims about important, or even critical underlying neuroanatomical sensorimotor structures for a concept with no obvious perceptual or action-based referent? Conceptual metaphor theory (Lakoff and Johnson, 1999) is often invoked to address this issue: central is the idea that we commonly talk, and importantly also think about relatively abstract domains (like time) in terms of more concrete domains (like space).

In order for embodied accounts of temporal conceptual representation to be either confirmed or disconfirmed first we must ask: What hypotheses about the neural instantiation of temporal concepts do metaphorical and simulation models generate? Although directed neural hypotheses are intuitive for the embodiment of concrete concepts - such as that of "apple" activating certain shapes, colors, and tastes - similar mappings onto sensory or 
action experiences are not transparent for more abstract concepts. So before clear-cut neural hypotheses can be formed, some foundational issues will need to be clarified. The present paper attempts to address issues related to the embodiment of temporal concepts.

We begin by reviewing the kinds of temporal concepts that tend to get mapped onto spatial relations. However, it is not clear to us that space, as relevant to the kinds of relations onto which temporal concepts are mapped, is itself embodied. A subsequent section therefore addresses the question of whether representations coding spatial concepts are best understood as embodied in the first place. Finally, we address the question of whether temporal concepts could be embodied more directly. Rather than making the claim that temporal concepts are embodied because they are mapped onto spatial concepts, one could ask if temporal concepts are embodied because they map onto sensory and motor representations of time itself. Perhaps embodied temporal concepts can bypass space. We briefly review temporal perception from a cognitive neuroscience perspective as relevant to embodied theories.

\section{SPACE, TIME, LANGUAGE, AND THOUGHT}

Conceptual metaphor theory (Lakoff and Johnson, 1999) provides a powerful framework for investigating the embodied representation of abstract concepts. At the core of conceptual metaphor theory is the idea that we commonly talk and think about relatively abstract domains (like time) in terms of more concrete domains (like space). Indeed, it seems as though spatial relations do provide structure for many abstract concepts. So, for example, we tend to conceptualize emotional states along a vertical axis (happy as up) and similarity in terms of proximity (difference as far; Meier and Robinson, 2004; Casasanto, 2008; Boot and Pecher, 2009). In this manner, "mental metaphors" (Casasanto, 2009) presumably help us to organize abstract concepts by mentally mapping a concept that we cannot easily perceive onto a concept more directly associated with perceptual or motor representations. These cross-domain mappings are not only argued to be conceptual in nature - i.e., more than mere linguistic artifacts of interest to etymologists - but they are argued to be asymmetrical - i.e., concrete concepts are thought to structure more abstract ones, but not vice versa. Experimental data collected using non-linguistic tasks bolster these claims. For example, Casasanto and Boroditsky (2008) found that the remembered size of a line in space concordantly modulates recall for its duration, but not vice versa. That is, (spatially) longer lines are remembered as being presented for longer times, but lines of greater durations are not remembered as having greater spatial extent. This, and other related findings (Boot and Pecher, 2009), are used to support the claim that patterns observed in language (i.e., the systematicity and asymmetry of space-time mappings) reflect deeper relations that influence other kinds of thinking. The spatial conceptualization of time has, in particular, been studied by both experimentalists and cognitive linguists in such great detail that time is postulated to be "the model system of choice for linguistic and psychological tests of relationships between metaphorical source and target domains" (Casasanto, 2009).

The model status of space-time relations owes something to the fact that conceptual divisions within the domain of space map intuitively onto complementary temporal concepts. For example, we talk about temporal extent or duration in terms of paths or distances in space (e.g., The Friday meeting marks the end of a long week); temporal order judgments, or sequences, in terms of dynamic object relations in space (e.g., The meeting comes before lunch); and the past and future in egocentric spatial terms (e.g., The meeting is behind us). Such a division of the time concept (duration; sequential order; past/future) seems to map onto traditional tripartite reference frame models that distinguish between extrinsic, intrinsic, and deictic frames of reference and also lend themselves to distinct spatial schemas (Kranjec, 2006; Zinken, 2009; Kranjec and McDonough, 2011; see Figures 1A-C).

Why are these relations between space and time so intuitive? Spatial and temporal relations share many similarities by being linked in experience. It does generally take more time to visually scan larger objects; objects that arrive earlier than other objects, in time, are generally in front of later objects, in space; and, as ambulatory, frontfacing organisms, events in our past tend to involve locations behind us. To some extent, this accounts for why temporal meanings can be easily expressed using spatial schemas. As we discuss in detail later, by spatial schemas we mean pared down, analog representations of spatial relations that can be depicted by points, lines, and vectors
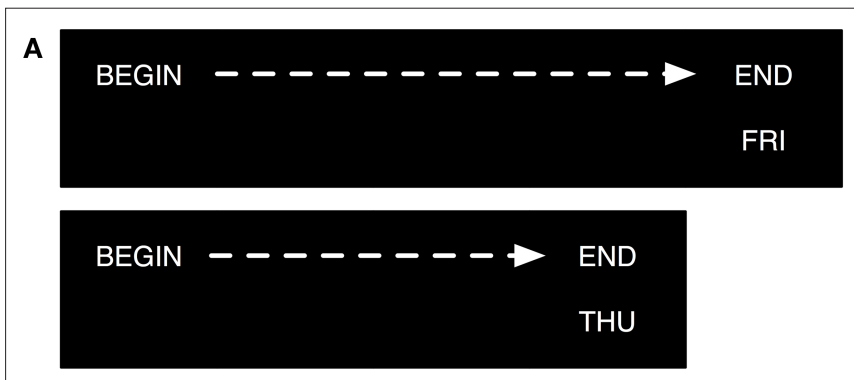

B

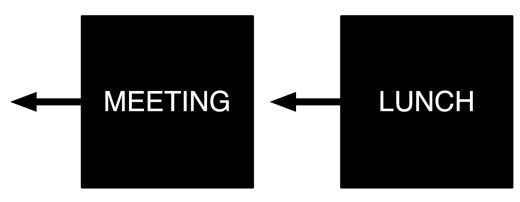

C

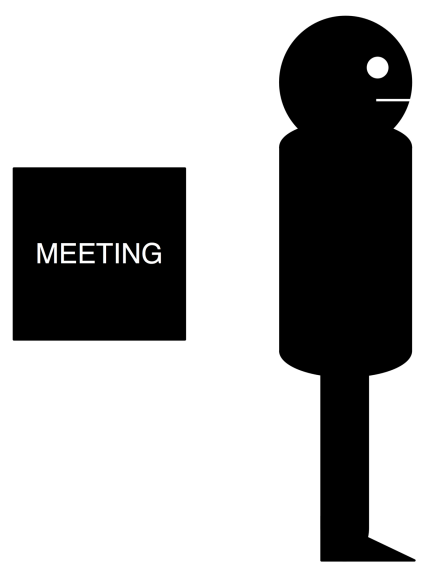

FIGURE 1 | Schematic depictions of three temporal concepts.

(A) Duration; A long work week ends Friday, a short one ends Thursday, (B) Sequence; The meeting comes before lunch, (C) Past/Future; The meeting is behind us. 
(Mandler, 1996; Talmy, 2000). The analog spatial characteristics of schemas would appear to map directly onto temporal phenomena. However, do these experiential-semantic correlations necessarily tell us that temporal concepts are embodied at the neural level?

\section{ABSTRACTING SPACE}

The idea that time is embodied because it is mapped onto space raises two fundamental questions; first, (1) how are spatial relations that are potentially important for structuring other concepts themselves represented in the brain and, second, (2) whether spatial relations represented as such are best understood as embodied. These two issues concern the representational continuity of spatial perception, thought, and language (Chatterjee, 2001, 2008). Roots of these ideas can be traced back to Pavio's (1986) dual coding hypothesis that suggested that information can be coded in analog as well as symbolic formats. Here, we are concerned with their interactions with respect to time and space and the ramifications for defining the limits of what can be reasonably considered an embodied abstract concept in the first place (Chatterjee, 2010). As things currently stand, the extent to which spatial relational information is in fact grounded in perceptual or sensorimotor content - i.e., spatial representation at the level described by embodied theories of cognition - is far from clear.

In conceptual metaphor theory, the idea of spatial relational schemas (Johnson, 1987; Mandler, 1992; Lakoff and Johnson, 1999; Talmy, 2000) plays a critical role in embodying abstract concepts. Schemas are generally described as "boiled down" abstractions of frequently observed spatial and motion patterns. Although they are often portrayed as static, iconic figures, or diagrams, mental schemas are better understood as multi or supramodal abstractions of frequently occurring patterns of movement, spatial relations, and force dynamics. Conceptual metaphor theory makes it clear that schemas are not the same as percepts ("We do not see spatial relations the way we see physical objects"; Lakoff and Johnson, 1999). Although their semantics often map onto the meanings of prepositions, they are thought to develop prior to word-like representations (Mandler, 1992). Schemas are also distinct from the kind of propositional or predicate logic structures that can operate on spatial relations in a mental model approach (Miller and JohnsonLaird, 1976) or the conceptual structures proposed by Jackendoff (1990). In many ways, schemas often seem to be defined in terms of what they are not, rather than what they are. Very broadly though, and the sense in which they are used in this paper, schemas can be understood as representations that code for abstract spatiotemporal relations among objects - like paths, containment, contact, and support relations - that provide a conceptual base onto which language can be mapped. They are not visual despite having analog properties; schemas represent the relations among objects, not the objects. They represent a pared down product of percepts, but not the percepts themselves (Mandler, 2000). Schemas therefore are generally treated as intermediate formats in multiple system approaches to mental representation (see Figure 2).

It is our view that the same basic spatial relation can be encoded in several formats, instantiated by distinct brain regions. Such a view may be compatible with other multiple system approaches. For example, Barsalou and colleagues propose that both words and perceptually grounded simulations play a role in representing concepts (Simmons et al., 2008); that supramodal representations of space and time serve an overarching role in structuring "perceptual symbols" (Barsalou, 1999); and that "relation simulators" serve as the mechanism for extracting abstract spatial and temporal relations from more perceptually rich imagistic representations (Barsalou, 2003). Kemmerer and Tranel (2000) found evidence for a double dissociation between linguistic and perceptual representations of spatial relations. A patient with left fronto-parietal damage did poorly on verbal tasks relying on categorical representations with relatively intact performance on coordinate visuospatial tasks, while a patient with damage to right frontal, parietal, and temporal areas displayed the opposite pattern. This suggests that abstract spatial relations may be stored separately in verbal and non-verbal formats.

Spatial schemas are, however, presently a theoretical construct. Although much has been written about schemas from a philosophical, developmental, and cognitive linguistic perspective, very little about their neural organization is understood. For present purposes, we can only review in more general terms how such abstract but discrete spatial relations, might be represented in the brain based on available neural data. In conducting research in this broader area, cognitive neuroscience has traditionally focused on (1) the left-hemisphere representation of spatial prepositions and categorical relations and (2) deficits in spatial representation associated with damage to the right-hemisphere (i.e., neglect).

For the current discussion, a short review of spatial prepositions and categorical relations in the context of cognitive neuroscience is a reasonable place to start. The spatial representation of time is

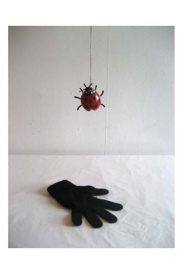

Scene

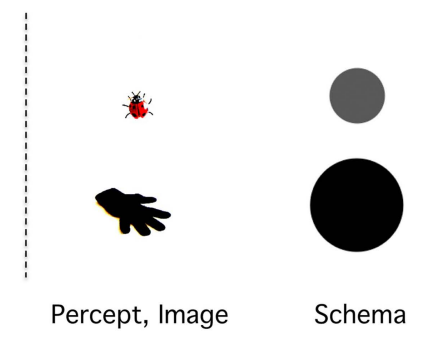

Percept, Image
[LOCATIVE RELATION] “above”

Conceptual structure analog structure. Conceptual structure is language-like, algebraic, and propositional. A verbal representation encodes the label referring to a discrete, categorical spatial relation (Adapted from Chatterjee, 2001).
FIGURE 2 |The mental representation of spatial relations. The perceptual or imagistic representation captures the sensory-rich features of the actual scene. The schema abstracts the relative positions of the objects while retaining some 
reliant on prepositions for expressing different kinds of temporal concepts. When communicating information about durations (e.g., We examined policy across the decade), sequences (The meeting was before lunch), and the past or future (The illness is far behind me) prepositions are used to invoke different kinds of spatial relations. Time concepts map onto spatial prepositions (Kranjec et al., 2010) and spatial prepositions, practically speaking, operate as relational schemas. For example, the preposition on can be used to represent the semantic relations between a pen on a table, and a boat on a lake. Similarly, when prepositions like on are used in the temporal domain as with, The meeting is on Wednesday, what is being invoked is a relational schema, emphasizing collocation, and contact.

Frederici (1981) demonstrated that aphasics with left posterior temporal-parietal lesions show semantic impairments that dissociate from syntactic ones in processing locative prepositions. Subsequently, Landau and Jackendoff (1993) proposed that the parietal cortex might process prepositions because it serves as the terminus of the dorsal "where" pathway. Damasio and colleagues (Damasio et al., 2001; Emmorey et al., 2002) corroborated this idea by finding a role for the left supramarginal gyrus and inferior frontal gyrus in the comprehension of locative prepositions. Noordzij et al. (2008) found that understanding the meanings of locative prepositions embedded in sentences, and the spatial relations expressed in picture formats were both associated with activation in the left supramarginal gyrus. And Wu et al. (2007) found that damage to the left inferior frontal-parietal cortices impaired knowledge of locative relations lexicalized by prepositions in simple sentences. In studies investigating categorical spatial relations using non-verbal tasks, additional work from our own laboratory and others also implicate left-hemisphere areas in the representation of the type of categorical spatial relations encoded by locative prepositions. Specifically, these studies find the inferior parietal lobe and frontal operculum to be involved (Kosslyn, 1987; Kosslyn et al., 1989, 1998; Amorapanth et al., 2010). The general view that emerges from both literatures - on spatial prepositions and on categorical spatial relations - suggests that the left-hemisphere, more than the right, processes these kinds of relations across verbal and non-verbal tasks. Furthermore, the inferior parietal cortex and possibly parts of dorsolateral prefrontal cortex involving inferior and posterior middle frontal gyri are critical in mediating this kind of spatial relational knowledge.

However, despite this left-hemisphere bias for processing prepositions and categorical spatial relations, spatial representation and spatial attention are more generally regarded as right-lateralized functions of the inferior parietal cortex, and the supramarginal gyrus in particular (McFie et al., 1950; Vallar and Perani, 1986). In contrast to left-hemisphere word-like representations, righthemisphere spatial representations are often described as being analog in format (Bisiach, 1993). Furthermore, representations of space in the right parietal lobe are thought to be supramodal, as there is evidence spatial deficits associated with neglect affect sensory modalities other than vision (Farah et al., 1989) and that right-hemisphere spatial representations are dissociable from visual imagery (Farah et al., 1988). Thus, spatial representation in the right-hemisphere can also be conceived as separable from any particular sensory modality. The picture that emerges from the cognitive neuroscience literature is one in which word-like spatial relations are represented in the left supramarginal gyrus and analog spatial relations are represented in the right supramarginal gyrus; where both kinds of representations may be more abstract than any particular sensory modality (see Figure 3).

The potential for abstract spatial relations to be instantiated in distinct formats of (1) amodal, left-hemisphere representations closely linked to language and/or (2) supramodal, right-hemisphere spatial representations linked more closely to perception, makes investigating image schemas with respect to sensorimotor grounding complicated. If representations of spatial relations provide organizational structure across sensorimotor modalities, how can one operationally distinguish between representations best characterized as either disembodied-and-amodal (in the left-hemisphere) or embodied-and-supramodal (in the right)? In fact, some philosophers think that making such an operational distinction between amodal and supramodal representations may be impossible (Dove, 2009; Machery, 2009). Barsalou (1999) states that supramodal spatial representations "constitute fundamental parts of perception that integrate...specific modalities (p. 638).” But if language itself is modeled as "part of perception" and also plays a role in "integrating modalities," the model has the risk of becoming circular; especially if spatial language is critical for providing structure across a range of abstract concepts. For example, some neo-Whorfian accounts suggest that verbal category labels exert an influence over perception and conceptualization (Lupyan, 2008; Regier and Kay, 2009; Lupyan et al., 2010). And it has been observed that patterns of spatial language exert an influence in both directions as well: over both time perception and abstract conceptualization (Boroditsky and Ramscar, 2002; Casasanto and Boroditsky, 2008). Although such work establishes that links exist between spatial language, perception, and the conceptualization of other domains - links that can persist even when non-linguistic tasks are employed - this work tells us less about the amodal, modal, or supramodal nature of the participating representations underlying a given behavioral effect.

While the research outlined above suggests that perceptual and conceptual systems are more integrated than previously thought, neural studies may permit researchers investigating space-time relations to make finer-grained comparisons of representations that serve as the basis for this integration. That is, by delineating the properties of potentially related, but distinct, mental representations in a multiple systems model, we can begin to ask if more word-like spatial labels or more percept-like image schemas ground temporal concepts. One step may involve determining if the neural instantiation of image schema-like spatial representations have more in common with the kinds of spatial relations thought to be instantiated in the left- or right-hemispheres. The more general challenge for cognitive neuroscience is to develop specific tasks and methods, such that, if there are discrete anatomical areas dedicated to processing the verbal, perceptual, or schematic representations of spatial relations, they can be identified. Once identified, the question would be: Do temporal concepts also engage the same neural structures?

Although empirical evidence for the existence of schematic representation is lacking, as a thought experiment, let us imagine that there was good functional and anatomical evidence available for both (1) the neural instantiation of schematic representations and specifically (2) the mapping of temporal relations onto structurally 
A

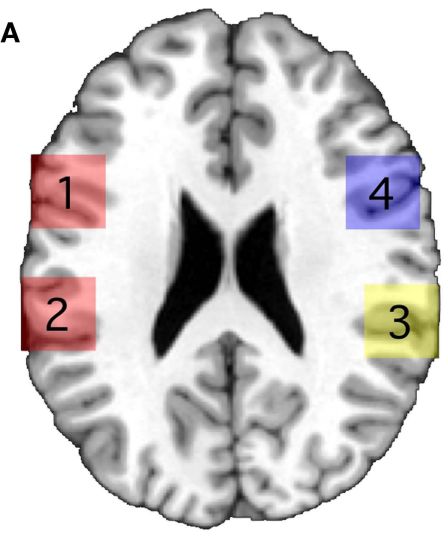

B

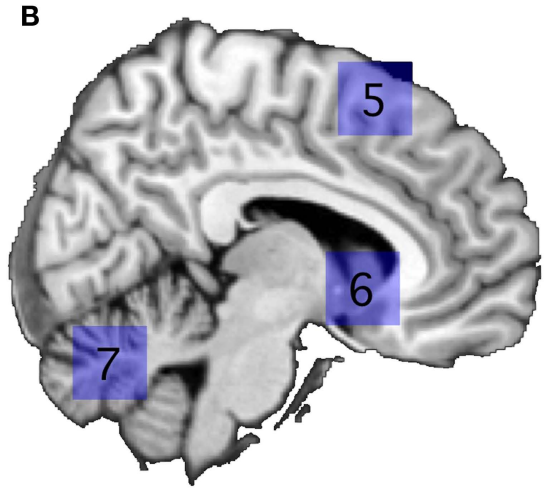

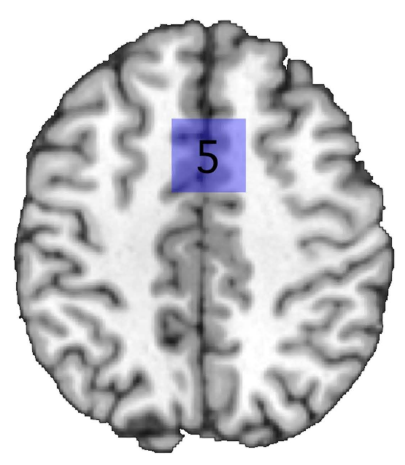

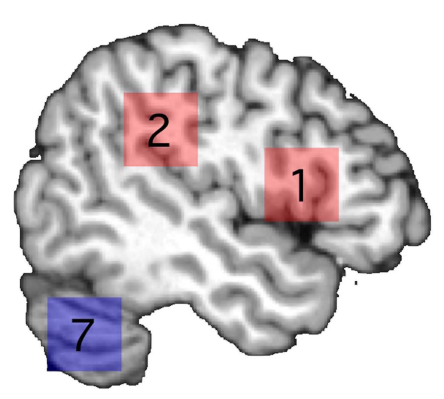

FIGURE 3 | (A) Transverse and (B) sagittal slices selected to illustrate anatomical areas discussed in Sections "Abstracting Space" and "Simulating Time" hypothesized to be important for locative and categorical spatial information (in red), supramodal spatial representation and attention (in yellow), and timing (in blue): Colored squares highlight areas including: (1) left inferior frontal gyrus, (2) left supramarginal gyrus, (3) right supramarginal gyrus, (4) right inferior frontal gyrus, (5) supplementary motor area, (6) basal ganglia (location of caudate shown here), and (7) cerebellum. concordant spatial schemas. The question would still remain: are schemas themselves best understood as embodied? In conceptual metaphor theory, schemas play a critical role in structuring more abstract concepts (like emotions, similarity, and time). However, since the idea of a schema remains a theoretical construct, it is particularly important to consider what finding schemas instantiated in the brain would actually tell us about embodiment.

Not much about schematic representation is agreed upon, but schemas, by most definitions, involve a process of moving away from the concrete perceptual attributes of objects in order to represent the more abstract relations between them. Thus, there are good reasons to hypothesize that representations resulting from such a process would in fact not involve perceptual or sensorimotor neural networks, or if they did, they would be greatly attenuated. In fact, the main proponents of embodied theories of mind acknowledge this limit. Lakoff (1987) writes that "image schemas are kinesthetic in nature, that is, they have to do with the sense of spatial locations, movement, shape, etc., independent of any particular sensory modality." (See Hampe, 2005 for more recent discussions, especially chapters by Dean and Grady). And Barsalou (2003) describes how abstract representations of spatial relations filter out the irrelevant details of rich simulations. Thus, even according to conceptual metaphor and simulation theories, when devising neural studies for tasks designed to invoke schemas, structures outside (but perhaps adjacent to) those sensorimotor areas thought to represent the most concrete aspects of direct sensory experience might be doing the most important work in representing abstract spatial relations. Using similar logic, stroke patients with lesions directly in perceptual or sensorimotor areas might be expected to retain the ability to represent the relations contained within perceptual arrays because those areas involved in fully representing the rich, perceptual features of visual displays may not be necessary for extracting the abstract relations between objects (in adult brains at least).

Considered from such a perspective, the role of schemas in a continuous, graded model of conceptual representation could actually be useful for marking where in this system embodiment trails off and abstraction begins. Before we can conclude one way or the other, many more details pertaining to how (and if) schematic relations are represented in the brain need to be known. Although empirical data relating to schematic representation is in short supply, we entertain the theoretical position that abstract, schemalike representations mediate between perception and language but that these representations are not embodied in the strict sense; i.e., there are no convincing reasons to think that schemas necessarily involve simulating previous experience with instances of a concept. According to our view, if schemas are instantiated in the brain they will be found to code the same kind of abstract structural roles 
among objects in space (and time) that are evoked by prepositions, but in a format and with a neural implementation that is distinct from both perceptual and verbal representations.

Conceived as such, schemas are disembodied at least as much as they are embodied. According to such a view one can, in principle, hold that schemas may be (a) derived from perceptual and motor experiences, (b) have some analog, and (c) some computational properties of abstract representations (as Barsalou, 1999 claims) and still maintain that such features are as characteristic of a "disembodied" representation as much as they are an "embodied" one. The notion that an abstract, analog representation need not be embodied in the strict simulation sense is the fulcrum of this position. It is an important point to consider because when one makes strong claims about the embodiment of image schemas one necessarily underplays the role of higher-order processes like relational thought, abstraction, and analogy that may play an especially important role during early cognitive development (Mandler, 2000; Gentner, 2003) and continue to mediate relations between spatial concepts and the more abstract concepts that find structure in space. Although not opposed to embodied cognition accounts, the view put forth here is compatible with a graded account of mental representation that resists making strong claims about the necessity of sensorimotor simulation in grounding language and thought (Chatterjee, 2010). It is also consistent with Talmy's (2000) view and Mandler's (2004) developmental framework which suggest that image schemas provide the foundation for explicitly accessible concepts. It is our position that conceptual meanings must be grounded on representations with content accessible to conscious analysis, thus it is unclear how sensorimotor representations impenetrable to conscious analysis could serve this purpose ${ }^{1}$. Schemas conceived in such a manner resemble the kind of meaningful but not perceptual right-hemisphere spatial representations described by early researchers of neglect:

\begin{abstract}
Spatial analogs may be claimed to be unfit to convey the full meaning a representation is supposed to be endowed with. In the absence of further comparative analysis of what is meant by "meaning" and "representation", my reply is that, on the one hand, meaning may be inherent in the kinetic features of spatial analogs (Bisiach and Berti, 1990), and, on the other, analogue representation may be conceived as being "enthymematic"- that is, leaving unexpressed a great deal of the antecedents and entailments of which meaning consists (Bisiach, 1992). Unlike pictures, as Sterelny (1990) would say, analogue representations are "preinterpreted" (Bisiach, 1993).
\end{abstract}

This view is consistent with the developmental mechanism that Mandler (2000) terms perceptual analysis and defines as "a process in which perceptual input is attentively analyzed and recoded into a new format." For schemas to be independently meaningful, they must come "preinterpreted."

\section{SIMULATING TIME}

But does an embodied temporal concept necessarily need a spatial schema for grounding? Consider the fact that we do not only talk about time in terms of space. We frequently talk about time in

${ }^{1}$ See Kranjec and McDonough (2011) for a thorough theoretical account for why image schema content should be expected to be explicit and therefore represented outside sensorimotor areas. We also report experimental evidence in support of this idea. its own terms; both grammatically and semantically. The focus on spatial metaphoric accounts of abstract conceptualization by embodied theories has had the ironic consequence of creating a blind spot for temporal language. By most accounts, the use of "purely" temporal, time-specific language precedes the use of metaphoric, spatial-temporal language in development (Nelson, 1996). Simulation approaches might closely examine how the brain codes obligatory grammatical categories like (viewer-centered) tense and (event-related) aspect, examining the ways in which such grammaticalization patterns could relate back to non-linguistic processes associated with the representation of distinct temporal reference frames. Similarly, the lexicalization of pure temporal concepts associated with particular temporal perspectives (e.g., egocentric: now, yesterday; duration: while, yet; sequence: later; frequency: always, never) also occurs sooner in development compared to spatial metaphoric language for time. Although little is known about the development of embodied concepts, the early acquisition of timepure lexical concepts and grammatical rule use implies, at least, that spatial grounding is not necessary for all temporal conceptualization. If space is not necessary for grounding temporal concepts, it suggests that support for simulation accounts might be found by looking for semantic grounding in sensorimotor networks associated with temporal, not spatial, processing.

The major claims made by simulation accounts of embodied cognition center around the basic idea that representing a particular concept at least partially entails the same sensorimotor neural networks activated during direct experience with the thing to which the concept refers. Recall that a general approach like this makes straightforward predictions. For example, representing concepts associated with actions (e.g., kick) should involve networks in primary motor cortex or visual motion areas, while representing concepts associated with sounds (e.g., thunder) should involve networks in primary auditory cortex. Predictions can be made with finer granularity within a domain such that an embodied hypothesis might predict that comprehending the meaning of punch would activate homuncular motor representations associated with arms more than legs, and kick, legs more than arms. If simulation accounts view concrete concepts as co-activating distributed networks of sensorimotor and domain-specific linguistic representations, how would one expect such a perspective to be applied to time? Although research on conceptual metaphor suggests that we look for the neural grounding of temporal concepts in representations of spatial schemas, as discussed above, neural structures supporting spatial schemas might not be sufficient to support simulation theories. Fortunately, the domain of time may permit a relatively direct approach for testing hypotheses about the embodiment of temporal concepts.

The brain has distinct neural mechanisms for representing different timescales and types of temporal information. Roughly, sub-second intervals are processed by subcortical areas, supra-second intervals by more diffuse cortical circuits, and the clock that underlies large-scale circadian rhythms is located in the suprachiasmatic nucleus of the hypothalamus (Buhusi and Meck, 2005). Recent meta-analyses further corroborate this view indicating that sub-cortical networks including the cerebellum and basal ganglia are strongly associated with processing the motor and perceptual components of sub-second timing tasks, while cortical areas like the supplementary motor area (SMA) and prefrontal cortex are more 
involved in supra-second timing (Wiener et al., 2010). However, a related lesion study suggests that the basal ganglia are not critical for timing per se, but rather with the production of timed movements (Coslett et al., 2010). Other recent work from our laboratory using $\mathrm{PMRI}$ and a duration discrimination task, also implicates the SMA in supra-second timing, in addition to left inferior frontal, and superior temporal cortical structures (Wencil et al., 2010). However, the perception of time is not simply a matter of representing duration. The neural instantiation of duration information dissociates with that for sequence information. There is evidence that making ordinal sequence judgments involves distinct premotor cortical areas as compared to making duration judgments (Schubotz and von Cramon, 2001) and that learning the sequence of a motor response involves right parietal structures while learning the duration of the same response, the cerebellum (Sakai et al., 2002). And although less accurately described as perceptual in nature, the neural architecture underlying thinking about the future and the past has also been investigated (Hassabis et al., 2007; Abraham et al., 2008; Arzy et al., 2009a,b). Other work finds the parietal cortex to be important for representing time in a more abstract sense of magnitude (Bueti and Walsh, 2009). Unlike other very abstract concepts (like hope for example), something is already known about the neural bases for several distinct cognitive processes associated with temporal cognition.

Considering what we know about timing in the brain, it seems that simulation approaches would benefit from using tasks and methods designed to probe within the domain of time to look for specific relations between neural areas specialized for a particular kind of time-specific cognitive processing (e.g., duration, sequence, or past/future representation) and an associated linguistic representation (e.g., distinctions between lexicalized concepts or grammatical class). If a simulation account of temporal conceptualization does not predict the grounding of time concepts in neural areas dedicated to processing temporal information it should explicitly state why this should be the case.

\section{CONCLUSIONS}

Time is the most frequently used noun in the English language (Soanes and Stevenson, 2007). But where do we look for temporal concepts in the brain? Current cognitive science approaches tend to investigate the spatial organization of temporal concepts and draw conclusions about embodied cognition. Although linguistic and experimental behavioral data are abundant, little neural data is available. This is not an ideal state for the field because providing direct evidence for the embodiment of abstract concepts is potentially possible within cognitive neuroscience (e.g., What are the neural bases for spatial schemas? For time perception? To what extent do time concepts map onto such structures?).

A study by Kemmerer (2005) is notable. In one of the few neuroscientific studies investigating the relations between space, time, and semantics, Kemmerer found evidence for a double dissociation for impaired comprehension of either the spatial or temporal meanings of prepositions in patients with focal brain lesions. The left supramarginal gyrus was determined to be a critical structure for representing the spatial meaning of prepositions, whereas areas important for representing temporal meanings were less clearly defined. However, the left perisylvian cortex was implicated. The results at least suggest that the spatial and temporal meanings of prepositions are represented separately, but are equivocal with respect to the current discussion. However, they suggest that the language of time is stored separately from the language of space. In general, embodied accounts would benefit by probing further for domain-specific dissociations between representations of temporal and spatial concepts in areas associated with spatial and temporal perception using cognitive neuroscience methods. Lesion studies, especially larger group studies, are particularly well suited for testing hypotheses about embodied cognition. They allow for making strong inferences regarding the necessity of a particular anatomical structure (e.g., perceptual or sensorimotor structures) for performing a given cognitive function. Well-designed lesion studies could provide especially valuable information concerning both (1) the roles that perceptual or schematic formats play in representing spatial relational information and (2) the part that such spatial representations vs. separate structures dedicated to temporal perception play in grounding our concepts of time (see Figure 3). Clearly, tasks that compare neural representations across verbal and perceptual tasks are required.

The ways in which we have described the conceptual grounding of time in either spatial abstractions or more directly in sensorimotor processes are not necessarily mutually exclusive. By probing domain-specific sensorimotor areas associated with temporal perception and perspective, cognitive neuroscientists place themselves in a position to potentially confirm aspects of simulation accounts while bypassing conceptual metaphor theories reliant on spatial schematic grounding. Alternatively, if temporal concepts find structure primarily in spatial abstractions, time could become the model domain for demonstrating how a particular abstract concept can become disembodied, or disconnected from the neural basis of its concrete representation rather than the other way around. The notion that conceptual processing involves both verbal and nonverbal representations is not new (Pavio, 1986) and has been refined in the context of more recent debates concerning embodiment and simulation theories (Louwerse, 2010; Riordan and Jones, 2010). In the case of space, distinguishing between formats presents a particular challenge, as it may be that the same basic spatial meanings encoded by amodal left-hemisphere verbal representations are also inherent to supramodal right-hemisphere representations.

Answers to questions related to these issues will depend partly on the neural evidence as it becomes available, but also on how theoretical discussions concerning the nature of spatial abstraction and conceptual scaffolding unfold. The present paper has tried to frame core issues concerning the embodied structure of abstract concepts with respect to some of what is currently known about the functional neuroanatomy of spatial and temporal representations. The depiction of embodied theories presented here may strike some as superficial. However, we are hopeful that the way common issues have been broadly framed will make addressing them more amenable to direct hypothesis testing using empirical methods. If timing areas play no role in grounding time concepts, those taking a strong embodied approach should be able to provide a rigorous explanation for why this is the case. If spatial representations ground time concepts, a more fine-grained neuroanatomical account capable of distinguishing between word-like representations in the left-hemisphere and more perceptual ones in the right-hemisphere may be possible. And finally, if the kinds of spatial schemas that are thought to ground temporal concepts cannot be easily identified, 
described, or contrasted with other kinds of spatial representations (i.e., symbolic or perceptual), then the validity of this proposed intermediate representational construct may need to be reconsidered by those taking an empirical approach.

\section{REFERENCES}

Abraham, A., Schubotz, R. I., and von Cramon, D.Y. (2008). Thinking about the future versus the past in personal and non-personal contexts. Brain Res. 1233, 106-119.

Amorapanth, P., Widick, P., and Chatterjee, A. (2010). The neural basis for spatial relations. J. Cogn. Neurosci. 8, 1739-1753.

Arévalo, A., Baldo, J., and Dronkers, N. (2010). What do brain lesions tell us about theories of embodied semantics and the human mirror neuron system? Cortex doi: 10.1016/j. cortex.2010.06.001. [Epub ahead of print].

Arzy, S., Bick, A., and Blanke, O. (2009a). Mental time in amnesia: evidence from bilateral medial temporal damage before and after recovery. Cogn. Neuropsychol. 26, 503-510.

Arzy, S., Collette, S., Ionta, S., Fornari, E., and Blanke, O. (2009b). Subjective mental time: the functional architecture of projecting the self to past and future. Eur. J. Neurosci. 30, 2009-2017.

Barsalou, L. W. (1999). Perceptual symbol systems. Behav. Brain Sci. 22, 577-660.

Barsalou, L. W. (2003). Abstraction in perceptual symbol systems. Phil. Trans. Roy. Soc. Lond. B Biol. Sci. 358, 1177-1187.

Bisiach, E. (1992). "Understanding consciousness," in The Neuropsychology of Consciousness, eds A. D. Milner and M. Rugg (London: Academic Press), 113-137.

Bisiach, E. (1993). Mental representation in unilateral neglect and related disorders: the twentieth Bartlett Memorial lecture. Q. J. Exp. Psychol. 46A, 435-461.

Bisiach, E., and Berti, A. (1990). "Waking images and neural activity," in The Psychophysiology of Mental Imagery, eds R. G. Kunzendorf and A. A. Sheikh (Amityville, NY: Baywood), 67-88.

Boot, I., and Pecher, D. (2009). Similarity is closeness: metaphorical mapping in a conceptual task. Q. J. Exp. Psychol. (Colchester) 63, 942-954.

Boroditsky, L. (2000). Metaphoric structuring: understanding time through spatial metaphors. Cognition 75, $1-28$.

Boroditsky, L. (2001). Does language shape thought? Mandarin and English speakers' conceptions of time. Cogn. Psychol. 43, 1-22.
Boroditsky, L., and Ramscar, M. (2002). The roles of body and mind in abstract thought. Psychol. Sci. 13, 185-189.

Buccino, G., Riggio, L., Melli, G., Binkofski, F., Gallese, V., and Rizzolatti, G. (2005). Listening to action-related sentences modulates the activity of the motor system: a combined TMS and behavioral study. Cogn. Brain Res. 24, 355-363. etal cortex and the representation of time, space, number and other magnitudes. Phil. Trans. Roy. Soc. B Biol. Sci. 364, 1831-1840.

Buhusi, C. V., and Meck, W. H. (2005). What makes us tick? Functional and neural mechanisms of interval timing. Nat. Rev. Neurosci. 6, 755-765.

Casasanto, D. (2008). Similarity and proximity: when does close in space mean close in mind? Mem. Cognit. 36, 1047-1056.

Casasanto, D. (2009). "When is a linguistic metaphor a conceptual metaphor?" in New Directions in Cognitive Linguistics, eds V. Evans and S. Pourcel (Amsterdam: John Benjamins), 127-145.

Casasanto, D., and Boroditsky, L. (2008). Time in the mind: using space to think about time. Cognition 106, 579-593.

Chatterjee,A. (2001). Language and space: some interactions. Trends Cogn. Sci. $5,55-61$.

Chatterjee, A. (2008). The neural organization of spatial thought and language. Semin. Speech Lang. 29, 226-238.

Chatterjee, A. (2010). Disembodying cognition. Lang. Cogn. 2, 79-116.

Clark, H. (1973). "Space, time, semantics and the child," in Cognitive Development and the Acquisition of Language, ed. T. Moore (New York: Academic Press).

Coslett, H. B., Wiener, M., and Chatterjee, A. (2010). Dissociable neural systems for timing: evidence from subjects with basal ganglia lesions. PLoS ONE 5, e10324. doi: 10.1371/journal. pone.0010324

Damasio, H., Grabowski, T. J., Tranel, D., Ponto, L.L.,Hichwa, R. D., and Damasio, A.R. (2001).Neural correlates of naming actions and of naming spatial relations. Neuroimage 13(Pt 1), 1053-1064.

Dove, G. (2009). Beyond perceptual symbols: a call for representational pluralism. Cognition 110, 412-431.

Emmorey, K., Damasio, H., McCullough, S., Grabowski, T., Ponto, L. L., Hichwa, R. D., and Bellugi, U. (2002). Neural
Bueti, D., and Walsh, V. (2009). The pari-

\section{ACKNOWLEDGMENTS}

This work was supported by the National Institutes of Health [RO1 DC004817, RO1 DC008779] and the National Science Foundation [subcontract under SBE0541957].

systems underlying spatial language in American sign language. Neuroimage $17,812-824$.

Evans, V. (2004). The Structure of Time: Language, Meaning and Temporal Cognition. Amsterdam: John Benjamins.

Farah, M. J., Hammond, K. M., Levine, D. N., and Calvanio, R. (1988).Visual and spatial mental-imagery - dissociable systems of representation. Cogn. Psychol. 20, 439-462.

Farah, M. J., Wong, A. B., Monheit, M. A., and Morrow, L. A. (1989). Parietal lobe mechanisms of spatial attention - modality-specific or supramodal. Neuropsychologia 27 461-470.

Frederici,A. (1981). Production and comprehension of prepositions in aphasia. Neuropsychologia 19, 191-199.

Gentner, D. (2003). "Why we're so smart," in Language in Mind, eds D. Gentner and S. Goldin-Meadows (Cambridge, MA: The MIT Press), 195-235.

Gentner, D., and Boroditsky, L. (2001). "Individuation, relativity and early word learning," in Language Acquisition and Conceptual Development, eds M. Bowerman and S. C. Levinson (England: Cambridge University Press), 215-256.

Glenberg, A. M., and Kaschak, M. P. (2002). Grounding language in action. Psychon. Bull. Rev. 9, 558-565.

Hampe, B. (2005). From Perception to Meaning: Image Schemas in Cognitive Linguistics, Vol. 29. Berlin: Mouton de Gruyter.

Hasplemath, M. (1997). From Space to Time: Temporal Adverbials in the World's Languages. Munich: Lincom Europa.

Hassabis, D., Kumaran, D., Vann, S. D. and Maguire, E. A. (2007). Patients with hippocampal amnesia cannot imagine new experiences. Proc. Natl. Acad. Sci. U.S.A. 104, 1726-1731.

Jackendoff, R. (1990). Semantic Structures. Cambridge, MA: The MIT Press.

Johnson, M. (1987). The Body in Mind. Chicago: Chicago University Press.

Kemmerer, D. (2005). The spatial and temporal meanings of English prepositions can be independently impaired. Neuropsychologia 44, 1607-1621.

Kemmerer, D., Castillo, J. G., Talavage, T., Patterson, S., and Wiley, C. (2008). Neuroanatomical distribution of five semantic components of verbs: evidence from fMRI. Brain Lang. 107, 16-43.
Kemmerer, D., and Tranel, D. (2000). A double dissociation between linguistic and perceptual representations of spatial relationships. Cogn. Neuropsychol. 17, 393-414.

Kosslyn, S. (1987). Seeing and imagining in the cerebral hemispheres: a computational analysis. Psychol. Rev. 94, 148-175.

Kosslyn, S., Koenig, O., Barrett, C., Cave, C., Tang, J., and Gabrielli, J. (1989). Evidence for two types of spatial representations: hemispheric specialization for categorical and coordinate relations. J. Exp. Psychol. Hum. Percept. Perform. 15, 723-735.

Kosslyn, S., Thompson, W., Gitelman, D., and Alpert, N. (1998). Neural systems that encode categorical versus coordinate spatial relations: PET investigations. Psychobiology 26, 333-347.

Kranjec, A. (2006). Extending spatial frames of reference to temporal concepts. Paper Presented at the TwentyEighth Annual Conference of the Cognitive Science Society, Vancouver.

Kranjec, A., Cardillo, E. R., Schmidt, G. L., and Chatterjee, A. (2010). Prescribed spatial prepositions influence how we think about time. Cognition 114, 111-116.

Kranjec, A., and McDonough, L. (2011). The implicit and explicit embodiment of time. J. Pragmat. 43, 735-748. (Special Issue: The Language of Space and Time).

Lakoff, G. (1987). Women, Fire, and Dangerous Things. Chicago: Chicago University Press.

Lakoff, G., and Johnson, M. (1999). Philosophy in the Flesh. New York, NY: Basic Books.

Landau, B., and Jackendoff, R. (1993). "What" and "where" in spatial language and spatial cognition. Behav. Brain Sci. 16, 217-265.

Louwerse, M. (2010). Symbol interdependency in symbolic and embodied cognition. Top. Cogn. Sci. doi: 10.1111/j.1756-8765.2010.01106.x. [Epub ahead of print].

Lupyan, G. (2008). The conceptual grouping effect: categories matter (and named categories matter more). Cognition 108, 566-577.

Lupyan, G., Thompson-Schill, S. L., and Swingley, D. (2010). Conceptual penetration of visual processing. Psychol. Sci. 21, 682-691.

Machery, E. (2009). Doing without Concepts. New York: Oxford University Press. 
Mahon, B. Z., and Caramazza, A. (2008). A critical look at the embodied cognition hypothesis and a new proposal for grounding conceptual content. J. Physiol. Paris 102, 59-70.

Mandler, J. M. (1992). How to build a baby: II. Conceptual primitives. Psychol. Rev. 99, 587-604.

Mandler,J.M. (1996). "Preverbal representation and language," in Language and Space, eds P. Bloom, M. A. Peterson, L. Nadel, and M. F. Garrett (Cambridge, MA: The MIT Press), 365-384.

Mandler, J.M. (2000). Perceptual and conceptual processes in infancy. J. Cogn. Dev. 1, 3-36.

Mandler, J. M. (2004). The Foundations of Mind: Origins of Conceptual Thought. New York, NY: Oxford University Press.

Matlock, T., Ramscar, M., and Boroditsky, L. (2005). On the experiential link between spatial and temporal language. Cogn. Sci. 29, 655-664.

McFie, J., Piercy, M. F., and Zangwill, O. L. (1950). Visual-spatial agnosia associated with lesions of the right cerebral hemisphere. Brain 73, 167-190.

Meier, B., and Robinson, M. (2004). Why the sunny side is up: associations between affect and vertical position. Psychol. Sci. 15, 243-247.

Miller, G., and Johnson-Laird, P. (1976). Language and Perception. Cambridge, MA: The Belknap Press of Harvard University Press.

Nelson, K. (1996). Language in Cognitive Development: Emergence of the Mediated Mind. Cambridge: Cambridge University Press.

Noordzij, M. L., Neggers, S. F. W., Ramsey, N. F., and Postma, A. (2008). Neural correlates of locative prepositions. Neuropsychologia 46, 1576-1580.
Nunez, R., Motz, B., and Teuschner, U. (2006). Time after time: the psychological reality of the ego-and time-reference-point distinction in metaphorical construals of time. Metaphor Symbol 21, 133-146.

Nunez, R., and Sweetser, E. (2006). With the future behind them: convergent evidence from Aymara language and gesture in the cross-linguistic comparison of spatial construals of time. Cogn. Sci. 30, 133-146.

Pavio, A. (1986). Mental Representations: A Dual Coding Approach. Oxford: Oxford University Press.

Pulvermuller, F., Hauk, O., Nikulin, V., and Ilmoniemi, R. J. (2005). Functional links between motor and language systems. Eur. J. Neurosci. 21, 793-797.

Regier, T., and Kay, P. (2009). Language, thought, and color: Whorf was half right. Trends Cogn. Sci. 13, 439-446.

Riordan, B., and Jones, M. N. (2010). Redundancy in perceptual and linguistic experience: comparing featurebased and distributional models of semantic representation. Top. Cogn. Sci. doi:10.1111/j.1756-8765.2010.01111.x. [Epub ahead of print].

Sakai, K., Ramnani, N., and Passingham, R. E. (2002). Learning of sequences of finger movements and timing: frontal lobe and action-oriented representation. J. Neurophysiol. 88, 2035-2046.

Santiago, J., Lupianez, J., Perez, E., and Funes, M. J. (2007). Time (also) flies from left to right. Psychon. Bull. Rev. $14,512-516$

Schubotz, R. I., and von Cramon, D. Y. (2001). Interval and ordinal properties of sequences are associated with distinct premotor areas. Cereb. Cortex 11,210-222.
Simmons, W. K.,Hamann, S. B.,Harenski, C. L., Hu, X. P., and Barsalou, L. W (2008). fMRI evidence for word association and situated simulation in conceptual processing. J. Physiol. Paris 102, 106-119.

Soanes, C., and Stevenson,A. (eds). (2007) Concise Oxford English Dictionary. Oxford, UK: Oxford University Press, 1728.

Sterelny, K. (1990). “The imagery debate," in Mind and Cognition, ed. W. G. Lycan (Cambridge, MA: Blackwell), 607-626.

Talmy, L. (2000). Towards a Cognitive Semantics: Concept Structuring Systems. Cambridge, MA: The MIT Press.

Tenbrink, T. (2007). Space, Time, and the use of Language: An Investigation of Relationships. Berlin: Mouton de Gruyter.

Teuscher, U., McQuire, M., Collins, J., and Coulson, S. (2008). Congruity effects in time and space: behavioral and ERP measures. Cogn. Sci. 32, 563-578.

Torralbo, A., Santiago, J., and Lupianez, J. (2006). Flexible conceptual projection of time onto spatial frames of reference. Cogn. Sci. 30, 745-757.

Vallar, G., and Perani, D. (1986). The anatomy of unilateral neglect after right-hemisphere stroke lesions - a clinical CT-scan correlation study in man. Neuropsychologia 24, 609-622.

Wencil, E. B., Coslett, H. B., Aguirre, J. K., and Chatterjee, A. (2010). Carving the clock at its component joints: neural bases for interval timing. $J$. Neurophysiol. 104, 160-168.

Wiener, M., Turkeltaub, P., and Coslett, H. B. (2010). The image of time: a voxel-wise meta-analysis. Neuroimage $49,1728-1740$.
Wilson, M. (2002). Six views of embodied cognition. Psychon. Bull. Rev. 9, 625-636.

Wu, D. H., Waller, S., and Chatterjee, A. (2007). The functional neuroanatomy of thematic role and locative relational knowledge. J. Cogn. Neurosci. 19, 1542-1555.

Zinken, J. (2009). “Temporal frames of reference," in Language, Cognition, and Space, eds P. Chilton and V. Evans (London: Equinox).

Zwaan, R.A. (2004)."The immersed experiencer: toward an embodied theory of language comprehension," in The Psychology of Learning and Motivation, Vol. 44, ed. B. H. Ross (New York: Academic Press), 35-62.

Conflict of Interest Statement: The authors declare that the research was conducted in the absence of any commercial or financial relationships that could be construed as a potential conflict of interest.

Received: 25 June 2010; accepted: 20 December 2010; published online: 31 December 2010

Citation: Kranjec A and Chatterjee A (2010) Are temporal concepts embodied? A challenge for cognitive neuroscience. Front. Psychology 1:240. doi: 10.3389/ fpsyg.2010.00240

This article was submitted to Frontiers in Cognition, a specialty of Frontiers in Psychology.

Copyright (c) 2010 Kranjec and Chatterjee. This is an open-access article subject to an exclusive license agreement between the authors and the Frontiers Research Foundation, which permits unrestricted use, distribution, and reproduction in any medium, provided the original authors and source are credited. 